\title{
Kämpferin gegen Geisterfahrer
}

Die funeralen Rituale waren in landesüblich würdiger Weise über die Bühne gegangen; selbst Herr Maurer von der SVP hatte sich herbeigelassen und Frau Dreifuss noch Respekt gezollt. Im Journal am Mittag hatten sich die Spitzen erleichtert und mit unverkennbarem Genuss ihrem Lieblingsthema zugewendet: der Personenspekulation bzw. der Nachfolgefrage. In der wahrscheinlich zunehmend präprandial bestimmten Diskussion war der Eifer erkennbar abgeebbt, als der Moderator die Teilnehmer mit der dringlichen Durchsage unterbrach, die Gefahr eines Geisterfahrers auf einem bündnerischen Autobahnabschnitt sei gebannt.

Das war analog zu Umberto Ecos Hyperrealität schon nahezu hypersymbolisch: Schweizerische Gesundheitspolitik nähert sich in der Tat immer mehr der Geisterfahrerei an, nur mit dem feinen Unterschied, dass sie nicht so einfach zu bannen ist. Frau Dreifuss hat mit Insistenz und Dossierkenntnis dieser Geisterfahrerei entgegenzuwirken versucht, teilweise mit Erfolg, häufig mit Misserfolg, wobei sie ihre persönliche wie politische Prinzipientreu ebenso motiviert wie gehemmt haben mag. Der grosse Wurf einer Sanierung der Gesundheitsfinanzierung bzw. des KVG ist ihr nicht gelungen, wobei anzufügen wäre, dass dies wohl keinem Sterblichen gegeben wäre, vor allem nicht in diesem Land. Gelingen kann dies nur in einem steten Vordringen, Wegmarke um Wegmarke; vielleicht wäre die Zahl der Wegmarken grösser gewesen, wenn sich Frau Dreifuss zur Analyse der Situation eine Brille mit ungetönten Brillengläsern aufgesetzt hätte, nicht eine mit rosa, nicht eine mit roten Brillengläsern (blau-gelbe Brillengläser standen nie zur Wahl).

La reine est morte, vive la reine (oder doch le roi)? Dies muss nicht unser Geschäft sein. Die Ärzteschaft hat mit jeder Magistratin, jedem Magistraten, den die Bundesversammlung wählt, sachlich und loyal zusammenzuarbeiten, sofern diese Spielregeln reziprok beachtet werden. Wir haben genügend damit zu tun, für eine moralisch integre wie sach- und fachkompetente Ärzteschaft uns ins Zeug zu legen; gelingt uns dies, dann haben wir keine Magistratin, keinen Magistraten zu fürchten.

Entwarnung vor Geisterfahrern kann auch damit nicht gegeben werden; dazu hat es noch zu viele Menschen auf der Strasse des Gesundheitswesens, die seltsame Fahrten unternehmen, sei es aus Unwissen oder verdecktem persönlichem Nutzen. Dies alles entbindet uns aber auch nicht von Selbstkritik: Wenn wir in Paraphrase des mehr als bekannten Witzes nur noch Geisterfahrer sehen, könnte das Problem auch auf unserer Seite liegen ...

Dr. med. Hans Heinrich Brunner FMH-Präsident 


\section{Un combat contre les risque-tout}

Les rites de départ ont été suivis de digne manière, comme il sied au pays. Même le tenant de l'UDC Ueli Maurer a usé de retenue et consenti à témoigner son respect à Mme Dreifuss. Au journal de midi, les chefs de file politiques, soulagés, n'ont pas attendu pour se consacrer avec un plaisir non dissimulé à leur sujet favori: les spéculations et guerres de succession. Dans la fièvre semble-t-il croissante animant les interlocuteurs, le meneur des débats annonce soudainement, à propos d'un automobiliste qui roulait à contresens sur l'autoroute, que tout danger avait été écarté.

Que voilà une scène hautement symbolique, à l'instar du style hyperréaliste d'Umberto Eco. La politique de santé suisse ressemble en effet de plus en plus à une course folle à contre-sens, avec la petite différence qu'elle n'est pas aussi facile que ça à arrêter. Mme Dreifuss a bien tenté, avec insistance et connaissance du dossier, de la freiner, parfois avec succès, parfois non, dans un esprit de fidélité à ses principes, personnels et politiques, qui a agi aussi bien comme frein que comme moteur. Son grand projet d'assainissement des finances de la santé, ou plus précisément de la LAMal, n'a pas abouti. Il faut bien reconnaître cependant qu'une telle entreprise est quasiment insurmontable, surtout dans un pays comme le nôtre, où l'on ne peut arriver à quelque chose que pas à pas, étape par étape. Il se peut que le nombre de ces étapes eût été plus grand si Mme Dreifuss avait usé de lunettes aux verres neutres, ni roses, ni rouges (une couleur plus «libérale» n'étant jamais entrée en ligne de compte!).

La reine est morte, vive la reine (ou le roi, qui sait?). Ce n'est pas à nous d'en décider. Le corps médical doit collaborer avec tout magistrat élu par l'Assemblée fédérale, loyalement, objectivement, pour autant que ces règles de bonne conduite soient respectées de part et d'autre. Nous avons suffisamment à faire pour nous battre en faveur d'un corps médical intègre, objectif et compétent. Y parviendrons-nous que nous n'aurons à craindre aucun magistrat.

La fin de l'alerte n'est pas donnée pour autant: nombreux sont encore ceux, dans le domaine de la santé publique, à prendre des voies étranges, par ignorance ou profit personnel. Que ce constat ne nous éloigne pas du sens de l'autocritique. En effet, si comme dans la plaisanterie bien connue, nous ne voyons plus que des automobilistes à contre-sens, il se pourrait que le problème soit aussi dans notre camp.

Dr Hans Heinrich Brunner président de la FMH 